\title{
ALLGEMEINE LITERATUR
}

F. Altermath, L'antithèse du corps psychique et du corps spirituel chez Irénée et chez Clément d'Alexandrie. Deux chapitres de l'histoire de l'exégèse de I Cor 15, 35-49: Resumé in Revue de Théologie et de Philosophie, Lausanne 1973, 394.

A. Benito y Duran, Las artes liberales en Clemente de Alejandría, in: Arts libéraux et philosophie au Moyen Age, Montreal-Paris 1969, 445-467.

J. Bernard, Die apologetische Methode bei Klemens von Alexandrien. Apologetik als Entfaltung der Theologie, Leipzig 1968 (Erfurter Theologische Studien 21).

W. den Boer, Some striking similarities in pagan and Christian allegorical interpretation, in: Studi filologici e storici in onore di V. de Falco, Napoli 1971, 465-473.

A. Böhlig, Zum Proverbientext des Clemens Alexandrinus, Byzantinische Forschungen (Amsterdam) 3, 1968, 73-79.

K. G. Bonis, Das humanistische Ideal nach Klemens von Alexandria, in: Proceedings of the first International Humanistic Symposium at Delphi I, Athen 1970, 211-225.

D. J. M. Bradley, The transformation of the Stoic ethic in Clement of Alexandria, Augustinianum 14, 1974, 41-66.

A. Brontesi, La soteria in Clemente Alessandrino, Rom 1972 (Analecta Gregoriana 186).

J. P. Broudéhoux, Mariage et famille chez Clément d'Alexandrie, Paris 1970 (Coll. Théologie historique 11).

A. Daniel, La cristologia di Paolo in Clemente Alessandrino, Studia Patavina 17, $1970,234-280$.

J. Daniélou, La tradition selon Clément d'Alexandrie, Augustinianum 12, 1972, 5-18.

A.-M. Denis, Introduction aux pseudépigraphes grecs d'Ancien Testament, Leiden 1970 (Studia in Veteris Testamenti Pseudepigrapha 1) (vgl. Index s. v. Clément d'Alexandrie).

B. D. Dupuy, Aux origines de la notion de théologie. Prophétie et théologie chez Clément d'Alexandrie, Mélanges Y. Congar, Paris 1974, 151-161.

G. Faggin, La pedagogia della patristica, in: La pedagogia, Storia e problemi, Milano 1971, 225-292. 
M. Farantos, Die Gerechtigkeit bei Klemens von Alexandrien, Diss. Bonn (1971) 1972.

W. E. G. Floyd, Clement of Alexandria's treatment of the problem of evil, Oxford 1971 (Oxford Theological Monographs).

V. Grossi, Un contributo agli studi su Clemente Alessandrino, Augustinianum 13, 1973, 149-152 (zu Lilla, s. u.).

A. Knauber, Die patrologische Schätzung des Clemens von Alexandrien bis zu seinem neuerlichen Bekanntwerden durch die ersten Druckeditionen des XVI. Jahrhunderts, in: Kyriakon, Festschrift für Johannes Quasten, herausg. v. P. GranfieldJ. A. Jungmann, I, Münster 1970, 289-309.

G. Ch. Koumakis, Das Sokratesbild in Therapeutik [!] des Theodoretus und seine Quellen, Platon 23, 1971, 337-351.

M. Kuyama, Pagan belief and Christianity in Clement of Alexandria, in: Memoirs of Kunitachi Music College 6, 1970, 51-63 (japanisch mit englischem Resumé).

M. Kuyama, Greek philosophy and Christian faith in Clement of Alexandria, Bulletin of the Society of Near Eastern Studies in Japan (Tokyo) 13, 1970, 109-127 (japanisch mit englischem Resumé).

S. R. C. Lilla, Clement of Alexandria. A study in Christian platonism and gnosticism, Oxford 1971 (Oxford Theological Monographs).

P. Maser, Die Siegelbildvorschläge des Clemens von Alexandrien und das spätantike rabbinische Judentum, Wiss. Zeitschrift der Universität Halle, Gesellschafts- und sprachwiss. Reihe 22, 1973, 65-70.

M. Mees, Die Zitate aus dem Neuen Testament bei Clemens von Alexandrien, Bari 1970 (Quaderni di Vetera Christianorum 2, urspr. Diss. Rom, Pontificio Istituto Biblico).

A. Méhat, Le lieu supracéleste de saint Justin à Origène, Studi in onore di M. Pellegrino, Torino $1975,282-294$.

B. Mondin, Il problema del linguaggio teologico dalle origini ad oggi, Brescia 1971 (Biblioteca di teologia contemporanea 8).

C. Moreschini, Luce e purificazione nella dottrina di Gregorio Nazianzeno, Augustinianum 13, 1973, 535-549.

R. Mortley, Connaissance religieuse et herméneutique chez Clément d'Alexandrie, Leiden 1973.

R. Mortley, The theme of silence in Clement of Alexandria, Journal of Theological Studies 24, 1973, 197-202.

R. Mortley, The mirror and I Cor. 13, 12 in the epistemology of Clement of Alexandria, Vigiliae Christianae 30, 1976, 109-120.

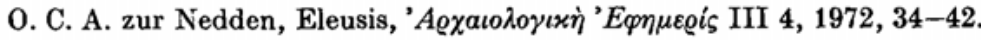

Madeline Nold, Alexandrian Christianity and Nag Hammadi religion, Studia Patristica XIV, Berlin 1976 (TU 117), 229-242.

D. Paulsen, Ethical individualism in Clement of Alexandria, Concordia Theological Monthly (St. Louis) 53, 1972, 3-20. 
H. Paulsen, Erwägungen zu Acta Apollonii 14-22, Zeitschrift f. d. neutestamentliche Wissenschaft 66, 1975, 117-126.

J. Pépin, La vraie dialectique selon Clément d'Alexandrie, in: Epektasis, Mélanges Jean Daniélou, Paris 1972, 375-383.

0. Prunet, La morale de Clément d'Alexandrie et le Nouveau Testament, Paris 1966 (Études d'histoire et de philosophie religieuses 61).

R. Riedinger, Der Physiologos und Klemens von Alexandreia, Byzantinische Zeitschrift 66, 1973, 273-307.

R. Riedinger, Zur antimarkionitischen Polemik des Klemens von Alexandreia, Vigiliae Christianae 29, 1975, 15-32.

A. M. Ritter, Christentum und Eigentum bei Klemens von Alexandrien auf dem Hintergrund der frühchristlichen Armenfrömmigkeit und der Ethik der kaiserzeitlichen Stoa, Zeitschrift f. Kirchengeschichte 86, 1975, 1-25.

M. Roncaglia, Méssage évangelique et humanisme hellénistique, I, Clément d'Alexandrie, Al-Machriq (Beyrouth) 63, 1969, 157-224.

M. Ruiz-Jurado, Le monde chez Clément d'Alexandrie, Revue d'histoire de la spiritualité 48, 1972, 5-23.

K. Schmoele, Läuterung nach dem Tode und pneumatische Auferstehung bei Klemens von Alexandrien, Münster 1974 (Münst. Beiträge zur Theologie 38).

E. Schrifner, „Gnostisches“ Christentum bei Clemens von Alexandrien. Untersuchung zum Glaubensverständnis in der alexandrinischen Kirche des Ausgehenden zweiten Jahrhunderts, Innsbruck 1969.

P. Schwanz, Imago Dei als christologisch-anthropologisches Problem in der Geschichte der Alten Kirche von Paulus bis Clemens von Alexandrien, Halle 1970 (Arbeiten zur Kirchengeschichte und Religionswissenschaft 2).

P. Schwanz, Der Wandel in der Gottesebenbildlichkeits-Vorstellung vom Neuen Testament zur frühen Patristik, Kairos 16, 1974, 268-294.

F. Solmsen, Early Christian interest in the theory of demonstration, in: Romanitas et Christianitas, Studia I. H. Waszink oblata, Amsterdam 1973, 281-291.

P. Stockmeier, Scheidung und Wiederverheiratung in der Alten Kirche, Theologische Quartalschrift 151, 1971, 39-51.

H. B. Timothy, The early Christian apologists and Greek philosophy exemplified by Irenaeus, Tertullian, and Clement of Alexandria, Assen 1973 (Philosophical Texts and Studies 21).

Y. Tissot, Hénogamie et remariage chez Clément d'Alexandrie: Resumé: Revue de Théologie et de Philosophie, Lausanne 1973, 395.

K. N. Tsirpanlis, The theology of history according to Clement of Alexandria, Platon 24, 1972, 252-274 (griechisch mit englischem Resumé).

F. K. Voros, The ethical fragments of Democritus. The problem of the authenticity,

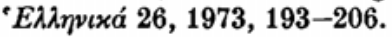


W. H. Wagner, A father's fate. Attitudes toward and interpretations of Clement of Alexandria, Journal of Religious History (Sydney) 6, 1971, 209-231.

G. Zaphiris, Le texte de l'Évangile selon saint Matthieu d'après les citations de Clément d'Alexandrie comparées aux citations des Pères et des théologiens grecs du $\mathrm{II}^{\mathrm{e}}$ au XVe siècle, Gembloux 1970.

\section{LITERATUR UND ADDENDA ZU BAND I}

Clemente Alessandrino, Il Protrettico, il Pedagogo. Introduzione, traduzione e commentario a cura di M. G. Bianco, Torino 1971 (Classici delle religioni. Sezione IV, La religione cattolica).

Paidagogos: Ausgewählte Schriften zur Pädagogik, besorgt von H. Kanz, Paderborn 1966.

G. Brambillasca, Citations de l'Écriture Sainte et des auteurs classiques dans le

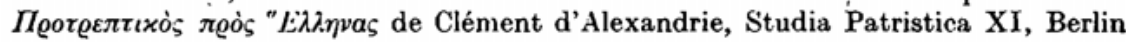
1972 (TU 108), 8-12.

A. H. C. van Eijk, The Gospel of Philip and Clement of Alexandria. Gnostic and ecclesiastical theology on the resurrection and the eucharist, Vigiliae Christianae 25, 1971, 94-120.

L. Alfonsi, Uno spunto posidiano in Clemente Alessandrino, in: Romanitas et Christianitas. Studia 1. H. Waszink oblata. Amsterdam 1973, 1-3.

7, 13-20 erhalten als Zitat im Cod. Ohrid, Nationalmuseum 84 (Mošin), 13. Jh.,

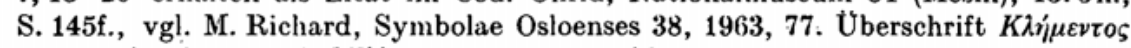

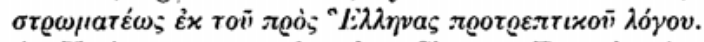

An Varianten gegenüber dem Clemens-Text der Ausgabe sind zu verzeichnen:

$\left.\left.13 \delta \iota^{\prime} \quad \ddot{v} v\right] \delta \iota \dot{o} \quad \vartheta \varepsilon o \bar{v}\right] \vartheta \varepsilon i o v 0$

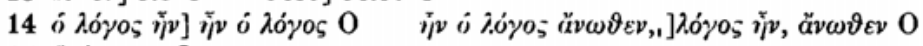

15 veía om. O

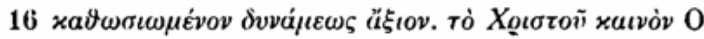

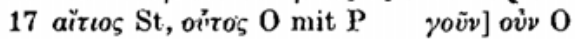

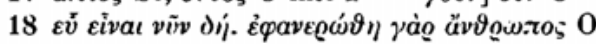

19 á $\pi \dot{\nu} \nu \tau \nu]$ ó $\pi \dot{\alpha} \nu \tau \omega \nu 0$

$51,10 \mathrm{vgl}$. L. Piccirilli, Susarione e la rivendicazione megarese dell'Origine della commedia greca, Annali della Scuola Normale Superiore di Pisa 4, 1974, 1289-1299.

61 vgl. F. J. Dölger, Das Sonnengleichnis in einer Weihnachtspredigt des Bischofs Zeno von Verona, Antike und Christentum 6, 1940, 4 u. ö.

68, 17 vgl. Q. Cataudella, Kahò $\dot{0}$ ※ivovvo , Sileno 1, Catania 1975, 23-36.

$68,19 f$. vgl. M. Tardieu, La lettre à Hipparque et les réminiscences pythagoriciennes de Clément d'Alexandrie, Vigiliae Christianae 28, 1974, 241-247, hier 246.

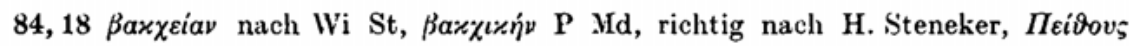

